\title{
Croissance de la pousse annuelle au cours des deux premières années du développement des rejets de châtaignier (Castanea sativa Mill). Mise en évidence d'un rythme endogène, influence du facteur thermique
}

\author{
JM Aufort*, M Carlué, JJ Fredon \\ Faculté des sciences, laboratoire de biologie végétale, 123, rue Albert-Thomas, \\ 87060 Limoges cedex, France
}

(reçu le 16 septembre 1988 ; accepté le 24 juin 1989)

\begin{abstract}
Résumé - L'étude a pour objectif de préciser la cinétique de la croissance de la pousse annuelle au cours des 2 premières années du développement des rejets de souche chez le châtaignier. L'analyse de la croissance résulte d'observations concernant l'allongement de la pousse, l'accroissement du diamètre à sa base, le nombre de feuilles. Les variations des différents paramètres sont comparées à celles de la température. L'allongement, l'épaississement et le nombre de feuilles ne varient pas de façon simultanée. Ainsi, la croissance en épaisseur de la pousse se poursuit après l'arrêt de l'allongement et de l'activité plastochronique. D'autre part, la durée de la période de croissance montre de fortes variations individuelles. Cependant, la croissance de la pousse s'établit le plus souvent en 2 phases principales au cours de la période d'activité annuelle. Ce comportement est particulièrement remarquable dans le cas de l'allongement où les 2 périodes d'activité sont séparées par une période de repos plus ou moins profond et prolongé. Parfois l'allongement se limite à la $1^{\text {re }}$ phase d'activité. Les caractéristiques de l'allongement d'une même pousse peuvent étre très différentes au cours des 2 phases d'activité, la longueur finale de la pousse étant mieux corellée avec l'allongement pendant la $2^{\text {nde }}$ phase que pendant la première. Enfin, c'est seulement au cours de la $1^{\text {re }}$ phase de l'allongement qu'on a pu mettre en évidence des correspondances entre les variations de la temperature et de la croissance.
\end{abstract}

Castanea sativa / rejet / pousse annuelle / phase de croissance / température

Summary - Growth of the annual sprout during the first two years in chestnut tree stump shoots (Castanea sativa Mili). The influence of temperature. The present investigation aims to clarify the growth kinetics of the annual sprout during the 1st 2 years in the development of chestnut stump shoots. The growth is evaluated from observations of elongation, diameter

\footnotetext{
* Correspondance et tirés à part
} 
growth, and number of leaves. The variations of these different parameters are compared with those of mean temperatures. The study of the sprout elongation during the 1st and the 2nd year reveals the presence of 2 phases of activity separated by deep and long rest periods. This takes place during late June or early July (figs 2a, 3a). A few sprouts stop lengthening at the end of the 1st active phase (fig 1a). The correlations between lengthening and temperatures are generally significant within the 1st active phase (fig 4) but non-significant within the 2 nd. The variations in the number of leaves (figs $1 b, 2 b$ ) correspond roughly to those of lengthening. However, the correlations between number of leaves and temperatures are seldomly significant (fig 5). The diameter growth of the sprouts also includes 2 main phases of activity, but the fluctuation periodicity is different from that of lengthening. In particular, the diameter growth continues after the lenghtening one (figs 1c, 2c, 3c). The correlations between diameter growth and temperature are never significant (fig 6). The growth of the annual sprout during the 1st 2 years in chestnut stump shoots is divided into 2 main phases of activity. However, the different parameters used to characterize the growth of the sprout do not vary simultaneously. The fluctuation in lengthening and the number of leaves seem to be synchronous, but this is not the case for diameter thickening. Finally, large individual variations can occur throughout the duration of the growing period and the growth velocity. For any given shoot, the characteristics of the 2 successive phases of activity can be very different so that the final size of the sprout correlates better with the growing characteristics during the 2nd phase rather than during the first.

\section{Castanea sativa / stump / annual sprout / growing phase / temperature}

\section{INTRODUCTION}

Dans les régions tempérées, l'élongation de la pousse annuelle d'un végétal ligneux peut présenter au cours de la période de végétation plusieurs phases d'activité séparées par de brèves périodes de repos. Dans le cas de vieux arbres on observe généralement une seule brève période d'allongement parfois suivie d'une $2^{\text {nde }}$ phase de croissance se manifestant sur un nombre limité de rameaux, tandis que, pour les plantules, on peut observer 3 ou 4 vagues successives d'activité (Zimmerman et Brown, 1971 ; Kramer et Kozlowski, 1979). Ce phénomène est également observé dans le cas de rejets de souche (Borchert, 1975 et 1976 ; Reich et al, 1980 ; Cobb et al, 1985).

Le déterminisme de cette croissance rythmique est mal connu. Certains considèrent que la $1^{\text {re }}$ phase de croissance correspondrait à l'allongement d'éléments préformés dans le bourgeon (Borchert, 1975 ; Zanette,
1981). L'influence de facteurs internes paraît évidente dans la mesure où des vagues successives de croissance peuvent se manifester dans un environnement dont les conditions restent constantes (Lavarenne-Allary, 1964 et 1966 ; Lavarenne et al, 1971 ; Zanette, 1981 ; Fromard, 1982).

Toutefois, I'influence des facteurs externes pourrait s'exercer en modulant la durée de chaque vague de croissance (Borchert, 1975) ou leur nombre (Lavarenne, 1966 ; Lavarenne et al, 1971). Chez le châtaignier, Si-Mohamed (1983) a montré que l'on pouvait observer une croissance rythmique chez les plantules maintenues en conditions constantes. Cependant, pour cette espèce fréquemment traitée en taillis, des observations similaires concernant les rejets de souche semblent manquer. Pourtant, certains travaux montrent que l'allongement des rejets n'est pas un phénomène uniforme et continu, Rullier-Breval (1985) a en effet constaté que le "maître rejet" d'une souche ne 
reste pas toujours le même au cours d'une saison de végétation.

Notre étude a donc pour objectif de préciser la dynamique de l'élongation de la pousse annuelle du châtaignier au cours des 2 premières années de la croissance des rejets de souche. Étant donné que la croissance d'un végétal ligneux comprend d'autres phénomènes que l'allongement, nous avons également recueilli des données concernant le plastochrone apparent et l'accroissement en épaisseur à la base de la pousse. L'approche de l'effet des facteurs externes a été limitée à celui de la température.

\section{MATÉRIEL ET MÉTHODES}

La parcelle de taillis de châtaignier utilisée se trouve sur la commune de Châlus, dans le Sud-Ouest du département de la HauteVienne. Cette région de collines, s'élevant jusqu'à une altitude d'environ $550 \mathrm{~m}$, jouit d'un climat à tendance océanique. Les précipitations sont abondantes, de l'ordre de $1200 \mathrm{~mm}$ par an en moyenne, sans saison sèche marquée (Vilks, 1974).

Les observations débutent en mai et s'achèvent en octobre 1983. Elles permettent d'apprécier la croissance des pousses qui se développent sur des souches recépées au cours de l'hiver précédent (pousse de $1^{\text {re }}$ année) et sur des rejets de 1 an (pousse de $2^{\text {nde }}$ année).

La température est relevée grâce à un thermometre enregistreur Richard et Pekly placé sur la parcelle expérimentale.

\section{Évaluation de la croissance des pousses}

La croissance des pousses est appréciée selon 3 critères : allongement, nombre de feuilles, augmentation du diamètre des pousses. On note également, pour chaque pousse, la date de la fin d'activité du bourgeon apical qui se manifeste par un jaunissement puis, plus ou moins rapidement, par la chute de l'extrémité de la tige.
L'intervalle de temps séparant 2 séries d'observations est d'une semaine. Les mesures sont réalisées sur un échantillon comprenant 25 pousses. Elles sont réparties sur 5 souches. Les 5 rejets choisis sont portés par des secteurs différents de la souche. Les longueurs sont mesurées à l'aide d'un mètre souple avec une précision de l'ordre du $\mathrm{cm}$. Le diametre est pris à la base de la pousse. On utilise un pied à coulisse électronique qui permet une précision de $0,1 \mathrm{~mm}$. Malgré les précautions prises (repérage du niveau de mesure, orientation de l'appareil) la précision n'est sans doute pas supérieure à $0,5 \mathrm{~mm}$.

Le nombre de feuilles est compté sans dissection du bourgeon. II correspond donc au plastochrone apparent qui n'exprime pas réellement la cinétique de formation des ébauches foliaires.

\section{Traitement des données numériques et présentation des résultats}

Pour l'étude de la croissance de la pousse de $1^{\text {re }}$ année, il n'est pas possible, au départ, de distinguer des rejets selon des classes de vigueur, car on ne connaît pas le devenir des bourgeons qui se développent sur la souche. Le choix est donc tout a fait aleatoire.

Pour l'étude des pousses de $2^{\text {nde }}$ année, nous avons donc été conduits à utiliser également un échantillon non homogène. Cependant, comme les brins les plus chétifs meurent très rapidement, ils ont été écartés. Ceux qui ont été choisis atteignaient au moins $1,30 \mathrm{~m}$ de longueur à la fin de la $1^{\text {ra }}$ année de croissance.

Étant donné l'hétérogénéité des échantillons, les moyennes perdent toute signification et risquent merme de masquer des phénomènes que peut révéler une analyse individuelle. C'est donc ce dernier type de démarche qui a été retenu.

Les résultats concernant la cinétique de l'allongement, de l'apparition des feuilles, de l'accroissement en diamètre sont présentés sous forme graphique. L'axe des abscisses indique une échelle de temps et porte la date des observations. Les paramètres permettant d'apprécier la croissance sont portés en ordonnée. Ce sont les valeurs différentielles qui sont prises en compte. Cette présentation permet de mettre en évi- 
dence les variations de la vitesse de croissance de façon plus nette qu'une courbe cumulative sur laquelle le ralentissement passager d'un phénomène se traduit seulement par un léger infléchissement de la pente.

Le tracé de ces courbes peut être comparé à celui de l'évolution des températures moyennes hebdomadaires.

Les coefficients de corrélations linéaires entre les variations de température et d'allongement sont calculés, pour chacune des phases du développement des pousses. Leur signification est estimée avec un degré de sécurité de $95 \%$.

\section{RÉSULTATS}

\section{L'allongement}

\section{Mise en évidence de 2 phases successives}

Malgré la grande variabilité des comportements individuels, l'examen des courbes d'allongement hebdomadaire des pousses suggere l'existence de 2 phases successives dans le temps, séparées par une période de ralentissement marqué. Au niveau d'analyse auquel nous nous situons il n'est pas possible de mettre en évidence un effet lié à la souche.

\section{Description de la $1^{r e}$ phase}

Elle se situe du début de la croissance des pousses, jusqu'à une période comprise entre le 22 juin et le 6 juillet. $\grave{A}$ ce moment là, certaines pousses cessent de s'accroître (fig ta) mais le plus souvent, l'allongement est très fortement ralenti (figs $2 a$ et $3 a$ ). Cette phase est caractérisée par la présence d'un maximum de l'allongement hebdomadaire aux alentours du 8 juin (figs $2 a$ et $3 a$ ). A la fin de cette première phase d'allongement, on peut

a

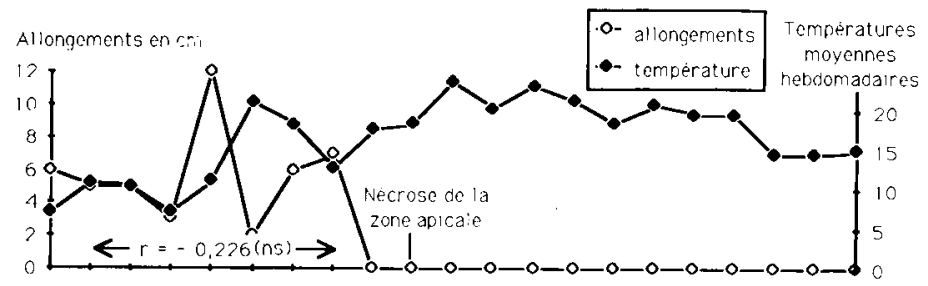

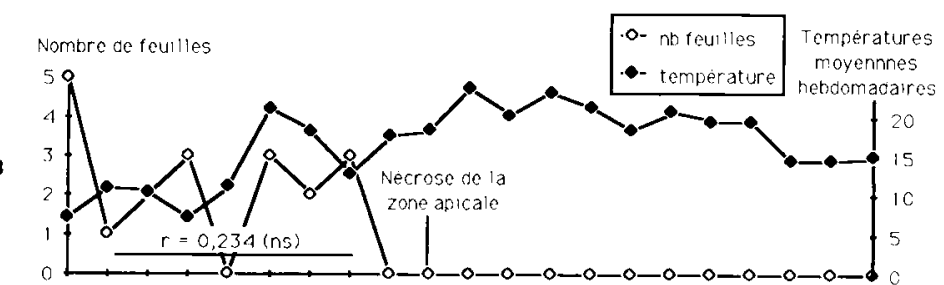

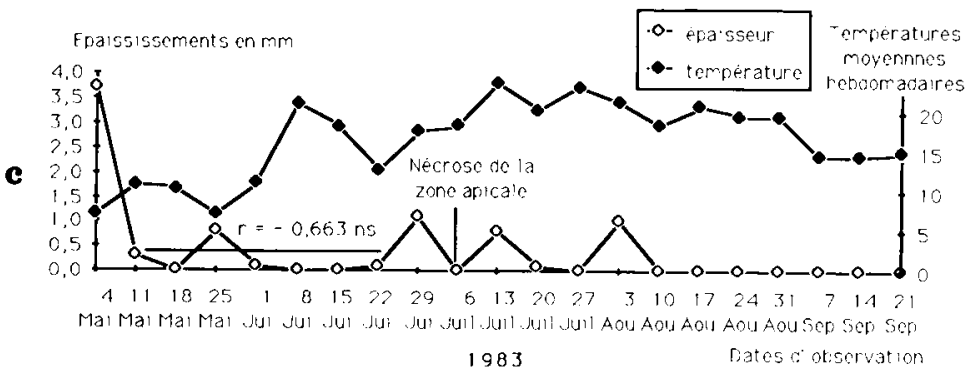

Fig 1 Rejet de $1^{\text {re }}$ année. Exemple d'un rejet qui perd précocement son bourgeon apical à ia fin de la $1^{\text {re }}$ phase de croissance et cesse définitivement de s'allonger. a : allongement ; b : nombre de teuilles: $\mathrm{C}$ : épaississement. 
relever différents types de comportement des pousses:

Nécrose, puis chute de la partie terminale (4 pousses de $1^{r e}$ année; 7 pousses de $2^{\text {nde }}$ année).

Ce phénomène n'est pas très fréquent à cette époque de l'année. II se manifeste sur les pousses les plus chétives ( 3 pousses de $1^{\text {re }}$ année mesurant moins de $49 \mathrm{~cm}$ et 2 pousses de $2^{\text {nde }}$ année mesurant moins de $39 \mathrm{~cm}$ ), mais aussi sur des pousses qui, selon ce critère, ne présentent aucune particularité remarquable.

\section{Arrêt de l'allongement}

Quelques pousses ne s'allongent plus pendant 1 ou 2 semaines (fig $2 a$ ).
Ralentissement très marqué de l'allongement

C'est le cas le plus fréquent. Le ralentissement peut être bref et n'apparaître qu'a l'occasion d'une seule observation hebdomadaire (fig $3 a$ ) ou se prolongera pendant 1 ou 2 semaines.

\section{Description de la deuxième phase}

Elle débute, selon les pousses, à la fin du mois de juin ou au début du mois de juillet. L'arrêt de l'allongement survient à des dates très variables allant, pour les pousses de $1^{\text {re }}$ année, depuis le 13 juillet jusqu'au 14 septembre et pour les pousses de $2^{\text {nde }}$ année, depuis le 29 juin jusqu'au 31 août. Mais dans la majorité des cas les pousses ont cessé de s'allonger le 17 août. En

$\mathbf{a}$

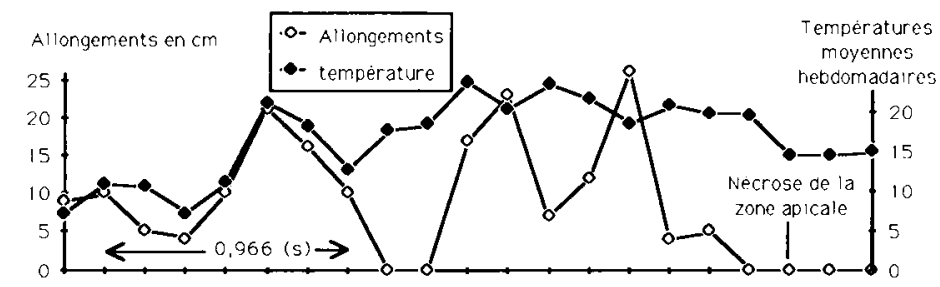

b
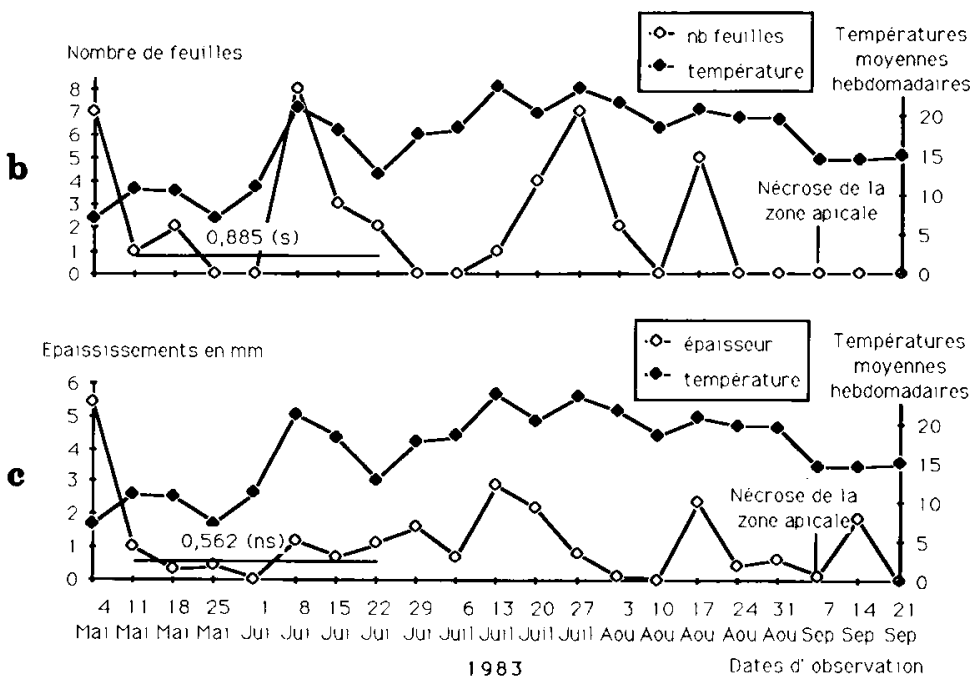

Fig 2 Rejet de $1^{\text {re }}$ année. Exemple d'un rejet qui cesse momentanément de s'allonger à la fin de la $1^{\text {re }}$ phase de croissance. Le bourgeon apical est conservé, mais se nécrose à la fin de la $2^{\text {ndo }}$ phase de croissance. $a$ : allongement; $b$ : nombre de feuilles, $c$ : épaississement. 

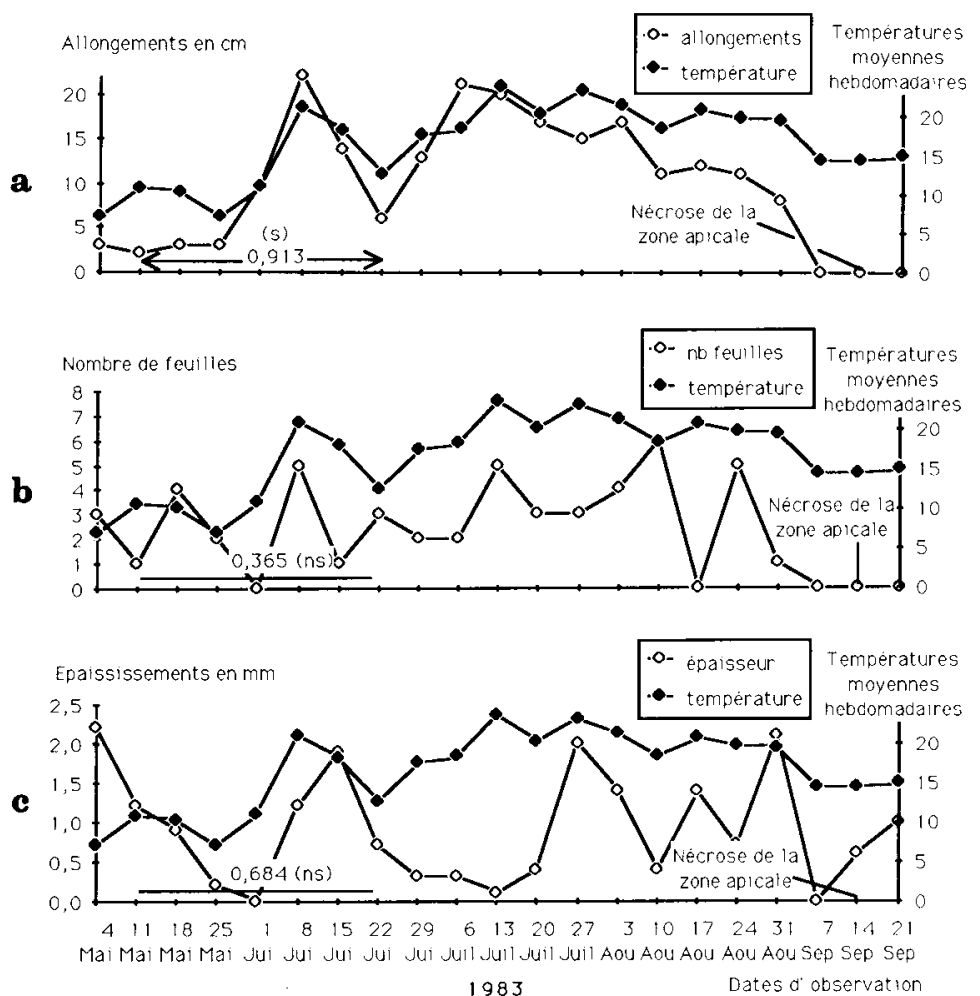

Fig 3 Rejet de $1^{\text {re }}$ année. Exemple d'un rejet pour lequel la fin de la $1^{r e}$ phase de croissance se manifeste par un ralentissement de l'allongement. La zone apicale se nécrose à la fin de la $2^{\text {nde }}$ phase de croissance. $a$ : allongement; $b$ : nombre de feuilles ; $c$ : épaississement.

fait, pour certaines pousses, cette phase d'allongement montre 2 à 3 périodes d'activité élevée séparées par des phases d'activité réduite (fig 2a). Ces périodes surviennent à des dates variables selon les individus. On peut distinguer plusieurs types de comportements :

- Lorsqu'à la fin de la $1^{\text {re }}$ phase de croissance, l'extrémité de la pousse n'est pas tombée, la reprise de l'allongement résulte de l'activité du bourgeon terminal. L'abscission de ce bourgeon, qui se manifestait avec une fréquence faible à la fin de la $1^{\text {re }}$ phase de l'allongement, devient quasi-générale. À la date où se terminent nos observations 5 pousses de $1^{\text {re }}$ année conservent leur bourgeon apical alors que toutes les pousses de $2^{\text {nde }}$ année l'ont alors perdu. La chute du bourgeon apical est observée en même temps que l'arrêt de la croissance en longueur ou dans un délai pouvant aller jusqu'à 4 semaines.

- Lorsque, à la fin de la $1^{\text {re }}$ phase d'allongement, le bourgeon terminal a disparu, certains rejets ne reprennent pas leur croissance (fig 1a), c'est le cas de 2 rejets de $1^{\text {re }}$ année et de 4 rejets de $2^{\text {nde }}$ année.

Chez les autres, le développement d'un bourgeon axillaire donne naissance à une pousse de longueur très inégale selon les rejets. Le nombre de cas observés reste trop faible pour qu'il soit possible de faire une analyse plus précise du phénomène. 
Relations entre allongement et température dans chacune des 2 phases de croissance

\section{Au cours de la $1^{r \theta}$ phase}

Les courbes de variation temporelle de la température et de l'allongement présentent un parallélisme évident (figs $2 a$ et $3 a$ ) a condition d'exclure quelques valeurs particulières (4 mai qui représente l'état initial et non l'accroissement entre 2 mesures et, dans certains cas, la valeur minimale de l'allongement hebdomadaire au moment du ralentissement de la croissance à la fin du mois de juin). Les corrélations allongement/température moyenne, sont le plus souvent significatives. C'est en effet le cas pour 19 rejets de $1^{\text {re }}$ année et pour 14 rejets de $2^{\text {nde }}$ année (fig 4).

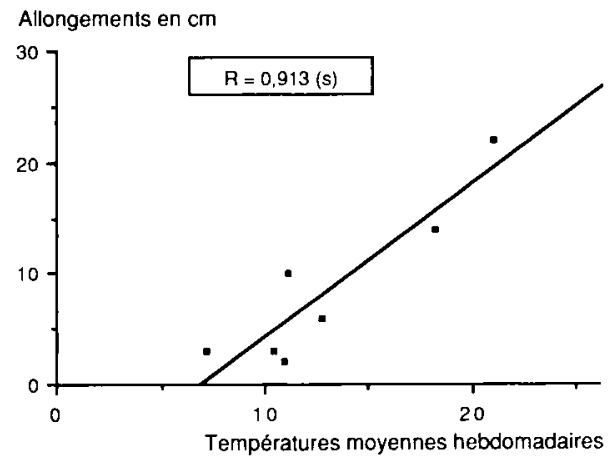

Fig 4 Rejet de $1^{\text {re }}$ année. Corrélations entre les températures moyennes hebdomadaires et l'allongement pendant la $1^{\text {re }}$ phase de croissance. $R$ : coefficient de corrélation. $S$ : significatif.

\section{Au cours de la deuxième phase}

Les variations de l'allongement ne correspondent pas toujours à des variations simultanées des valeurs moyennes hebdomadaires de la température. En particulier, l'arrêt de croissance se fait alors que les températures se maintiennent encore à un niveau élevé. Pour l'ensemble de cette phase, les corrélations existant entre les valeurs moyennes des températures et l'allongement ne sont jamais significatives dans le cas des pousses de $2^{\text {nde }}$ année et très rarement (7 cas) dans le cas des pousses de $1^{\text {re }}$ année.

\section{Relations entre la longueur finale des pousses et leur allongement au cours de chacune des 2 phases de croissance}

II s'établit une relation significative entre la longueur finale des pousses et leur longueur à la fin de la $1^{\text {re }}$ phase d'allongement. Toutefois cette relation n'est pas très étroite $(r=0,762$ dans le cas des pousses de $1^{\text {re }}$ année ; $r=0,466$ dans le cas des pousses de $2^{\text {nde }}$ année). Une relation bien plus étroite s'établit entre la longueur finale des pousses et leur allongement pendant la $2^{\text {nde }}$ phase de croissance $(r=0,978$ dans le cas des pousses de première année ; $x=0,950$ dans le cas des pousses de $2^{\text {nde }}$ année).

Dans les 2 cas il apparaît donc que l'accroissement de la pousse pendant la $2^{\text {nde }}$ phase de l'allongement a une importance particulière dans le déterminisme de sa longueur finale. On peut en effet remarquer que le comportement des pousses peut varier considérablement d'une phase à l'autre. C'est par exemple le cas de 2 pousses de $1^{\text {re }}$ année qui, à la fin de la $1^{\text {re }}$ phase de croissance, ont une longueur voisine $(63$ et $65 \mathrm{~cm}$ ) mais qui s'allongent l'une de $45 \mathrm{~cm}$, l'autre de $57 \mathrm{~cm}$ pendant la $2^{\text {nde }}$ phase.

Quatre des pousses de $2^{\text {nde }}$ année qui à la fin de la $1^{\text {re }}$ phase mesurent $59,61,61$ et $60 \mathrm{~cm}$ s'accroissent respectivement de $28,35,65$ et $89 \mathrm{~cm}$ pendant la $2^{\text {nde }}$ phase. Deux pousses qui, à la fin de la $1^{\text {re }}$ phase, se classent 
parmi les plus courtes ( 34 et $37 \mathrm{~cm}$ ) s'allongent respectivement de 71 et 79 $\mathrm{cm}$ au cours de la seconde.

\section{Le plastochrone apparent}

\section{Description des courbes}

Les pousses de $1^{\text {re }}$ et de $2^{\text {nde }}$ année ont un comportement voisin. Le tracé des courbes représentant l'évolution temporelle du nombre de feuilles (figs $1 b, 2 b, 3 b)$ montre une activité maximale au début du mois de juin. La période de la fin du mois de juin ou du début du mois de juillet correspond à une phase d'activité ralentie. Elle reprend pendant les mois de juillet et d'août mais l'apparition de nouvelles feuilles est alors très irrégulière. La seule différence notable concerne le début de la période de végétation où, pendant le mois de mai, l'accroissement du nombre de feuilles est plus rapide pour les pousses de $2^{\text {nde }}$ année que pour les pousses de $1^{\text {re }}$ année. Lorsque l'extrémité de la pousse tombe les premiers signes de la nécrose se manifestent entre 2 et 5 semaines après l'arrêt du développement des feuilles.

\section{Relations entre le nombre de feuilles et l'allongement}

Le tracé des courbes représentant l'évolution temporelle de l'apparition des feuilles montre visiblement quelques analogies avec celui de l'allongement. Dans les 2 cas le début du mois de juin correspond à une période d'activité importante (figs $1 b, 2 b, 3 b$ ). Cependant les maxima pour l'apparition des feuilles précèdent parfois d'une semaine ceux de l'allongement.
Une période d'activité ralentie s'observe à la fin du mois de juin ou au début du mois de juillet (figs $2 b, 3 b$ ).

L'arrêt de l'apparition des feuilles est assez souvent noté en même temps que celui de l'allongement ( 12 cas pour les pousses de $1^{\text {re }}$ année, 7 cas pour les pousses de $2^{\text {nde }}$ année) mais aussi avec 1 ou 3 semaines d'avance sur l'arrêt de l'allongement (fig 2b).

Cependant, dans le cas des pousses de $2^{\text {nde }}$ année, l'apparition d'un nombre important de feuilles au début du mois de mai ne correspond pas à un allongement rapide de la pousse car les entre-nœuds formés à ce moment restent tres courts.

D'autre part il existe une relation significative entre le nombre total de feuilles et la longueur finale de la pousse $(r=0,899$ dans le cas des pousses de $1^{\text {re }}$ année ; $r=0,936$ dans le cas des pousses de $2^{\text {nde }}$ année). $\mathrm{Si}$ on prend comme référence les 2 phases définies pour la croissance en longueur, la relation entre l'allongement et le nombre de feuilles est meilleure pendant la $2^{\text {nde }}$ phase que pendant la $1^{\text {re }}$ (cas des pousses de $1^{\text {re }}$ année: $1^{\text {re }}$ phase $r=0,809,2^{\text {nde }}$ phase $r=0,839$ : cas des pousses de $2^{\text {nde }}$ année : $1^{\text {re }}$ phase $r=0,885$; $2^{\text {nde }}$ phase $r=0,928$ ).

\section{Relations entre le nombre de feuilles et les températures moyennes heb- domadaires}

Bien que la liaison entre l'allongement et le nombre de feuilles paraisse assez étroite, les corrélations significatives entre l'apparition des feuilles et les températures moyennes hebdomadaires sont exceptionnelles ( 1 cas au cours de la $1^{\text {re }}$ phase d'allongement et 3 cas au cours de la $2^{\text {nde }}$ phase pour les pousses de $1^{\text {re }}$ année). Les corré- 
lations sont le plus souvent peu étroites (fig 5). Cette contradiction apparente résulte sans doute du fait que l'on mesure un phénomène discontinu et que l'effet de la température se manifeste avec un certain retard.

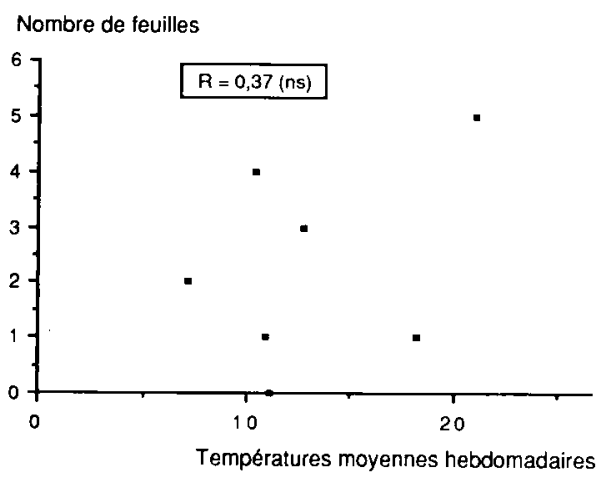

Fig 5 Rejet de $1^{\text {re }}$ année. Corrélations entre les températures moyennes hebdomadaires et le nombre de feuilles pendant la $1^{\text {re }}$ phase de croissance. $R$ : coefficient de corrélation. NS : non significatif.

\section{La croissance en épaisseur}

\section{Description des courbes}

L'accroissement en diamètre est d'abord toujours lent. II s'affaiblit encore dans la période correspondant à la fin de la $1^{\text {re }}$ phase d'allongement (figs $2 \mathrm{c}$ et $3 c$ ). II est plus actif au cours des mois de juillet août et septembre et se poursuit assez tard (figs $2 \mathrm{c}$ et $3 \mathrm{c}$ ) après l'arrêt de l'allongement. Dans le cas des pousses de $1^{\text {re }}$ année l'arrêt de la croissance en épaisseur est observé 1 semaine (5 pousses) 2 semaines ( 4 pousses, fig 2c) 3 semaines ( 3 pousses, fig $3 c$ ) et même 5 semaines ( 2 pousses) après l'arrêt de l'allongement. Pour 7 pousses l'accroissement en diamètre n'a pas cessé lorsque s'achève les observations. Pour les pousses de $2^{\text {nde }}$ année le délai est de
4 semaines ( 6 pousses), 5 semaines (4 pousses), 6 semaines ( 4 pousses), 7 semaines ( 3 pousses) et même 10 semaines (2 pousses). Un accroissement radial tardif peut même se manifester pour les pousses qui ne s'allongent plus après l'abscission précoce de leur bourgeon apical (fig 1c).

\section{Relations entre croissance en épais- seur et allongement}

Manifestement, les périodes d'activité pour l'allongement et la croissance en épaisseur n'apparaissent pas synchrones même si dans les 2 cas on observe une periode de repos plus ou moins profond à la fin du mois de juin ou au début du mois de juillet.

Cependant il s'établit une relation significative entre le diamètre final à la base des rejets et leur longueur ( $r=0,899$ dans le cas des pousses de $1^{\text {re }}$ année; $r=0,880$ dans le cas des pousses de $2^{\text {nde }}$ année). De même, si on prend pour référence les phases de croissance definies pour l'allongement, les corrélations entre diamètre et longueur des rejets à la fin de la $1^{\text {re }}$ phase (pousses de $1^{\text {re }}$ année $r=0,861$; pousses de $2^{\text {nde }}$ année $r=0,834$ ) et croissance diametrale et allongement pendant la $2^{\text {nde }}$ phase (pousses de $1^{\text {re }}$ année $r=0,861$; pousses de $2^{\text {nde }}$ année $r=0,706$ ) sont également significatives.

\section{Relations entre la croissance en épaisseur et les températures moyennes hebdomadaires}

Les relations entre les valeurs moyennes hebdomadaires de la température et l'accroissement en épaisseur des pousses ne sont jamais significatives, aussi bien pendant la $1^{\text {re }}$ phase que pendant la $2^{\text {nde }}$ phase d'allonge- 
ment (fig 6). Les fluctuations de la croissance diamétrale des pousses semblent donc dépendre, soit de facteurs externes autres que la temperature, soit de facteurs endogènes.

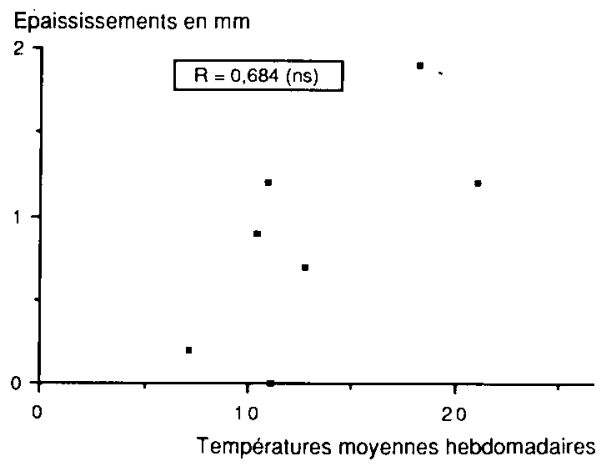

Fig 6 Rejet de $1^{\text {re }}$ année. Corrélations entre les températures moyennes hebdomadaires et l'epaississement a la base de la pousse pendant la $1^{\text {re }}$ phase de croissance. $\mathrm{R}$ : coefficient de corrélation. NS : non significatif.

\section{DISCUSSION ET CONCLUSION}

Ces observations montrent que les différents paramètres utilisés pour caractériser la croissance de la pousse de l'année des jeunes rejets de châtaignier, ne varient pas de façon simultanée.

Les fluctuations de l'apparition des feuilles et de l'allongement apparaissent assez bien synchrones mais diffèrent nettement de celles de l'accroissement en diamètre. II est bien connu que chez les ligneux l'arrêt de l'apparition des feuilles précède nettement celui de l'épaississement. Dans le cas des rejets du châtaignier, ce phénomène est également observé par Berthier (1984) et Rullier-Breval (1985). On peut remarquer qu'un accroissement en diametre se manifeste chez des pousses particulièrement chétives, qui paraissent en état de repos profond (allongement et apparition des feuilles nuls, extrémité de la tige nécrosée). La durée de la période de croissance de la pousse montre de fortes variations individuelles. La variabilité de la durée de période de croissance des rejets de souche a été observée pour de nombreuses especes, notamment par Lavarenne-Allary (1964) chez $P$ mnus spinosa - $C$ rataegus monogyna et oxyacantha - Tilia platyphylbs Rham nus fiangula - Sorbus term nalis et $C$ anpinus betula. II en est de même pour les plantules des espèces ligneuses comme le signalent Klebs (1914) et Fromard (1982) sur le hêtre, Lavarenne-Allary (1966) sur le chêne et Zanette (1981) sur le pommier.

II apparaît surtout que la croissance de la pousse s'établit en 2 phases principales au cours de la période d'activité annuelle.

C'est pour l'allongement que ces 2 phases d'activité se remarquent le mieux, car au phénomène physiologique d'arrêt de croissance, correspond un signal morphologique, la nécrose de l'extrémité de certaines pousses.

- La fin de la $1^{\text {re }}$ phase de croissance survient à la fin du mois de juin ou au début de celui de juillet, dans un intervalle de temps très réduit même s'il n'y a pas une évolution rigoureusement synchrone de toutes les pousses. II ne semble pas que ces variations puissent résulter d'un effet lié à la souche. À cette période de l'année correspond également, pour le plastochrone apparent et l'accroissement en diamètre, une phase d'activité ralentie. Une inhibition d'origine endogène pourrait se manifester à ce moment, non seulement sur l'allongement mais aussi sur le plastochrone et l'épaississement.

- Après une période de repos plus ou moins marquée, s'installe une $2^{\text {nde }}$ va- 
gue de croissance dont la durée est très variable selon les individus. La fin de cette période d'activité se manifeste généralement par la chute de la partie terminale de la pousse.

- Les dimensions finales d'une pousse dépendent bien sûr des caractéristiques de chaque vague de croissance. Mais il faut souligner l'importance tout a fait particuliere de la $2^{\text {nde }}$. En effet, quel que soit le phénomène analysé, on trouve toujours une meilleure corrélation pour croissance finale/croissance réalisée pendant la $2^{\text {nde }}$ phase que pour croissance finale/croissance réalisée pendant la $1^{\text {re }}$ phase. Cela montre également que, au moins pendant les 2 premières années du développement des rejets, les corrélations qui peuvent se manifester au sein d'une cépée pendant la période annuelle de croissance ne sont pas immuables. Des fluctuations imprévisibles peuvent apparaître et rendent aléatoires des prévisions sur l'avenir des rejets, obtenues en fonction d'observations ponctuelles.

La croissance en longueur des rejets est bien correlée aux variations de la température pendant la $1^{\text {re }}$ phase de l'allongement.

Le fait qu'elle ne le soit pas au cours de la $2^{\text {nde }}$ phase résulte probablement de l'arrêt de la croissance à une époque où les températures sont encore élevées. - Mais un autre effet des variations de la température pourrait correspondre à une modulation de la durée des vagues de croissance. Si-Mohamed (1983) a en effet observé que la durée des vagues de croissance varie pour des plantules cultivées en conditions naturelles selon les annees, ou pour des plantules cultivées en conditions constantes selon la température. On peut s'interroger sur le rôle de la baisse de temperature observée entre le 8 et le 22 juillet. Elle entraîne un ralentisse- ment de la vitesse d'élongation de la pousse mais elle pourrait également favoriser ou provoquer l'installation d'une inhibition plus ou moins profonde des processus de croissance. II est en effet remarquable de constater que la fin de la $1^{\text {re }}$ vague de croissance intervient pour toutes les pousses dans un laps de temps réduit, comme sous l'influence d'un signal indépendant des individus ou des groupes d'individus appartenant à une même souche.

- Si les variations du facteur thermique peuvent avoir un effet régulateur sur la durée des différentes vagues de croissance, l'existence même d'un rythme dépend de facteurs endogènes. De nombreuses hypothèses ont été proposées. Borchert (1975) et Zanette (1981) rappellent une hypothèse fréquemment avancée pour expliquer l'allongement en 2 phases de la pousse des végétaux ligneux des régions tempérées pendant la période annuelle d'activité. La $1^{\text {re }}$ phase pourrait correspondre à l'allongement des organes préformés dans le bourgeon, la $2^{\text {nde }}$ à celle des organes néoformés. Chez le châtaignier, à la fin de la $1^{\text {re }}$ phase de croissance, les pousses de $1^{\text {re }}$ année portent de 13 à 25 feuilles, celles de $2^{\text {nde }}$ année en ont de 11 à 24. Si on compare ces chiffres à ceux donnés par Assaf (1966) qui compte entre 5 et 7 ébauches foliaires dans un bourgeon de châtaignier 3 semaines avant le débourrement, l'activité organogène serait alors très importante pendant la $1^{\text {re }}$ phase de l'allongement. Toutefois, le nombre d'ébauches préformées pendant la $1^{\text {re }}$ phase est certainement moindre que celui résultant des chiffres données par Assaf (1966). En effet cet auteur étudie des bourgeons d'arbres fruitiers. Quelques observations nous ont montré que les bourgeons dormants du sommet de jeunes pousses 
de taillis contiennent entre 10 et 14 ébauches foliaires. II y aurait donc, pour les pousses de $2^{\text {nde }}$ année, néoformation d'une dizaine d'ébauches pendant la $1^{\text {re }}$ phase de croissance. Le cas des pousses de $1^{\text {re }}$ année est plus difficile à cerner car on ne connait rien de l'ébauche à partir de laquelle se développe la pousse (organes préformés enfouis sous le rhytidome, bourgeons de néoformation au niveau du bourrelet cambial ?). On peut penser que l'activité organogène pendant la $1^{\text {re }}$ phase de croissance est ici encore plus importante que pour les pousses de $2^{\text {nde }}$ année. Quoiqu'il en soit, la $1^{\text {re }}$ vague d'allongement ne peut résulter, du moins dans le cas du châtaignier, de la seule croissance des organes préformés dans le bourgeon. Pendant cette période, l'organogenèse n'est pas négligeable mais nous ignorons si elle suit des fluctuations comparables à celles de l'allongement ou du dégagement des feuilles.

Les observations effectuées au cours de ce travail ne permettent pas une analyse plus approfondie des causes de la succession de vagues de croissance pendant la période de végétation annuelle.

Comme pour bien d'autres espèces ligneuses des régions tempérées, ce phénomène se manifeste pendant les premières années de l'édification des pousses des rejets du châtaignier. Une meilleure compréhension des mécanismes ne pourrait être apportée que par une étude physiologique et dynamique des corrélations qui s'exercent entre les rejets portés par une même souche.

\section{REMERCIEMENTS}

Les auteurs adressent leurs vifs remerciements à Monsieur JC Mauget, direc- teur du laboratoire de bioclimatologie à I'INRA de Clermont-Ferrand pour ses critiques et suggestions.

\section{RÉFÉRENCES}

Assaf R (1966) Étude des bourgeons végétatifs de quelques espèces fruitières. J Agric Trop Bot AppI 4-5, 139-146

Berthier E (1984) Fonctionnement d'un écosystème forestier : croissance, biomasse et productivité du compartiment ligneux épigé des taillis de châtaignier (Castanea sativa $M$ ill du sud-est de la France. Thèse de $3^{e}$ cycle, Grenoble, $62 \mathrm{pp}$

Borchert R. (1975) Endogenous shoot growth rhythms and indeterminate shoot growth in oak. Physiolp lant, 35, 152-157

Borchert R (1976) Differences in shoot growth patterns between juvenile and adult trees and their interpretation based on system analysis of trees. Acta Hort; $56,123-130$

Cobb SW, Miller AE, Zahner R (1985) Recurrent shoot flushes in scarlet oak stump sprouts. For Sci 31, 725-730

Fromard L (1982) Croissance rythmique et variabilité chez le hêtre ( $F$ agus sylvatica L). DEA, Clermont-Ferrand $11,56 \mathrm{pp}$

Klebs G (1914) Über das Treiben der einheimischen Bäume, speziell des Buche, Abh Heideberg, Akad W iss, 3, 1-113

Kramer PJ, Kozlowski $\Pi$ (1979) Physiobgy of woody plants. Academic Press New York, $811 \mathrm{pp}$

Lavarenne-Allary S (1964) Recherches sur la croissance des bourgeons de chêne et de quelques autres especes ligneuses. These Doctorat d'État, Clermont-Ferrand, 200 pp

Lavarenne-Allary S (1966) Croissance rythmique de quelques espèces de chêne cultivées en chambre climatisées. $C R$ Acad Sciparis, SérD, 262, 358-361

Lavarenne $S$, Champagnat $P$, Barnola $P$ (1971) Croissance rythmique de quelques végétaux ligneux de régions tempérées cultivés en chambres climatisées à température élevée et constante et sous diverses photopériodes. BuIlsoc Bot Fr 118, 131-162 
Reich PB, Teskey RO, Johnson PS, Hinckley TM (1980) Periodic root and shoot growth in oak. For Sci 26, 590-598

Rullier-Breval B (1985) Croissance d'un taillis de châtaignier aprés coupe. Étude au cours des trois premieres années. Thèse Doctorat d'Université Paris XI, $155 \mathrm{pp}$

Si-Mohamed C (1983) Germination ; rythmes de croissance et morphogenese de jeunes plants chez Castanea sativa $M$ iller Thèse Doctorat $3^{\theta}$ cycle, ClermontFerrand II, $200 \mathrm{pp}$
Vilks A (1974) Contribution à l'étude phytogéographique du département de la Haute-Vienne. Thèse Doctorat $3^{\theta}$ cycle, Toulouse, $127 \mathrm{pp}$

Zanette F (1981) Recherches descriptives et expérimentales sur la morphogenèse des systèmes aériens et racinaires de quelques porte-greffes de pommiers. Thèse Docteur Ingénieur, Clermont-Ferrand II, $159 \mathrm{pp}$

Zimmerman $\mathrm{MH}$, Brown CL (1971) Trees : structure and function. Springer Verlag, New York, $336 \mathrm{pp}$ 